\title{
Experimental and CFD Modelling of the Drift Flux in Two-Phase Air- (Non) Newtonian Slug-Flow Pattern Flow along Horizontal and Inclined Pipelines
}

\author{
Paula D. Pico ${ }^{1}$, Juan P. Valdés ${ }^{1}$, Nicolás Ratkovich ${ }^{1}$, Eduardo Pereyra ${ }^{2}$ \\ ${ }^{1}$ Department of Chemical Engineering, Universidad de los Andes \\ Carrera 1 \# 18a-12 Bogota, Colombia \\ pd.pico10@uniandes.edu.co; jp.valdes10@uniandes.edu.co; n.rios262@uniandes.edu.co \\ ${ }^{2} \mathrm{McD}$ ougall School of Petroleum Engineering, The University of Tulsa \\ Tulsa, OK 74104, United States \\ eduardo-pereyra@utulsa.edu
}

\begin{abstract}
The present study analyzes the influence of the physical properties of Newtonian and non-Newtonian fluids, such as density, effective viscosity and surface tension, as well as operational parameters of the piping, such as diameter, length and angle of inclination, on the drift velocity for two-phase gas-liquid flow. This study comprises experimental and Computational Fluid Dynamic (CFD) approaches. The simulation model consists of half a section of a circular pipe with a symmetry plane. This model was calibrated through a mesh independence test, which considered experimental and literature data as benchmarking values for both low and high viscosity Newtonian fluids. The results obtained through the CFD model showed good agreement with the experimental data gathered for the present study, keeping the experimental deviations under 30\% for all cases considered. The relationship between the Froude number (Fr) and the Viscosity number (Nvis) was studied and an inverse exponential tendency was found for all the parameters and fluids tested, which agrees with the models proposed in literature. The data gathered for all fluids on the drift velocity's behavior against operational parameters such as length, diameter and inclination, showed the influence of the governing forces for each case based on dimensionless analysis using Eötvos (Eö) and Reynolds (Re) numbers. For dominant capillary or viscous forces on the system, the drift velocity changed with the variation of these parameters. However, it was found that for dominant inertial and gravitational forces, the drift velocity maintained a constant value regardless of the operational settings. Finally, it was observed that the rheological nature found for the non-Newtonian fluids has a significant influence on the drift velocity's behavior, deviating its patterns from the Newtonian fluids as the effective viscosity changed.
\end{abstract}

Keywords: Drift Flux, CFD, Froude Number, Viscosity Number, Slug flow, Two-phase, Benjamin Bubble.

\section{Introduction}

In the past few decades, all efforts of the oil and gas industry have shifted towards the extraction, transportation and refinement of heavier oils, as they represent nearly $70 \%$ of the actual available reserves of crude oil [1]. In the past, all crude oil extraction was focused almost exclusively on light oil reserves, as the exploration and drilling technologies were only available for low-viscosity fluids, which meant lesser costs associated with its production. However, these reserves were depleted, which led to the need of increasing and improving extraction technologies for high density and viscosity hydrocarbons. Non-Newtonian fluids are also commonly encountered in the upstream petroleum industry as waxy crude oils, gelled oils, drilling muds, fracturing fluids (for non-conventional oil and gas resources) and slurries [2]. Therefore, it became of great interest to extend the applicability range and improve the existing knowledge on multiphase flow models to consider liquid phases with high viscosities and Non-Newtonian rheological behavior.

Currently, most of the existing multiphase flow models used to predict important operational parameters, such as translational velocity of the oil mixture (translational velocity) or pressure drop along the pipeline, are only accurate for low viscosity fluids $\left(<0.01 \mathrm{~Pa}^{*} \mathrm{~s}\right)$ and have a wide range of limitations to be properly applied. Consequently, these models do not account for the effects of high viscosity or apparent viscosity (for Non-Newtonian fluids) and cannot be generalized for various operational conditions and neither can be unified for variations on fluid properties. Moreover, few different approaches can be found in literature for slug flow pattern modelling and they present incomplete or narrow-scoped 
correlations to estimate important parameters such as the drift flux. Various studies, Gregory \& Scott (1969) [3], Duckler \& Hubbard (1975) [4] and Heywood \& Richardson (1979) [5] initially considered a null drift velocity in the estimation for the translational velocity of the slug unit in horizontal or near horizontal pipes. However, it has been demonstrated analytically, Benjamin (1968) [6] and Weber (1981) [7], and experimentally, Zukoski (1966) [8], Bendiksen (1984) [9] and Weber et al. (1986) [10] that the drift velocity component is not zero, even for a horizontal arrangement of the pipeline. Based on that, drift velocity should be accounted for in the estimation of the translational velocity for slug flow.

Several researchers have recently taken the study of multiphase flow transport phenomena to Computation Fluid Dynamics (CFD) simulations. This has generated an increment and improvement of the data available to guarantee an efficient, reliable and accurate prediction of two-phase flow behavior on pipelines without the need to spend on expensive experimental facilities and tests. There are several approaches for the computational modelling of two-phase flow behavior. The most common approaches include Eulerian-Eulerian multiphase method and Volume of Fluid (VOF) method, the latter being the selected method on this research. The present study will be focusing on the analysis of the drift flux behavior for Newtonian and Non-Newtonian fluids on horizontal and inclined pipelines, considering two-phase, gas-liquid slug flow using CFD software STAR-CCM+. This investigation will provide a relationship between a series of physical and operational parameters considered with the drift velocity using quantitative and qualitative dimensionless analysis between Froude number (Fr), Viscosity number (Nvis), Reynolds number (Re) and the Eötvos number (Eö).

\subsection{Translational Velocity}

The term translational velocity for multiphase flow studies is defined as the velocity at which a slug unit travels [1]. The slug unit refers to the combination of gas bubbles travelling along with alternating liquid slugs, generating the commonly known slug flow pattern. This velocity is usually expressed in terms of the velocity component of the total mixture multiplied by a flow coefficient or distribution parameter and the drift velocity. The initial expression for the translational velocity of the slug unit was proposed by Nicklin et al. (1962) [11] as shown in Eq (1).

$$
v_{T}=C_{o} v_{m}+v_{d}
$$

The distribution parameter $\left(C_{0}\right)$ is defined as the approximate ratio between the maximum and the average velocity of the slug unit considering a fully developed velocity profile. This ratio is determined from the assumption that the propagation velocity of the gas bubble follows the maximum local liquid velocity in front of the nose tip as proposed by Kroes \& Henkes (2014) [12]. Current multiphase flow models use the translational velocity to understand the behavior of the mixture travelling along the pipeline. To properly calculate this parameter, it is important to consider the contribution made by the drift velocity.

\subsection{Drift Velocity}

The drift flux parameter refers to the velocity at which the gaseous phase travels and penetrates through the stagnant liquid phase within the pipe. The drift flux model treats the mixture as a single pseudo-fluid rather than two separate phases, yet it considers the interface slip and interactions between both phases [13]. Consequently, the drift flux model consists of only four field differential equations from the original six, eliminating one energy and one momentum equation. Given this, it is important to mention that the drift flux model replaces dynamic interactions (relative motion and energy difference) between phases given by the field functions with constitutive laws which provide relative velocity between phases (kinematic relation between phases) and thermal interaction between phases [14]. The differential equations by which the drift flux is modelled include continuity for one phase (usually the gaseous phase) and three conservation equations for the mixture which include continuity, momentum and energy [14]. Based on the formulation mentioned, the drift-flux model follows the standard approach used to analyze the dynamics of a mixture of gases or miscible liquids. Therefore, this model is generally well accepted for mixtures where the dynamics of both phases are closely coupled and share a well-defined interphase surface region.

This model takes into account the effects of non-uniform velocity and void fraction profiles, as well as the effect of the local relative velocity between the phases [15]. Its application has resulted very successful in several engineering problems related with forced convection systems involving two-phase flow dynamics. The drift flux model however results much simpler in its formulation in comparison to other two-fluid models due to several considerable assumptions that must 
be considered, one of them being the pseudo-fluid treatment for the two-phase mixture. On the other hand, in the application of engineering problems, these assumptions become very useful as they allow detailed analysis of two-phase flow behavior to be carried out with less difficulty. In two-phase flow dynamics, information required for engineering problems usually comes from the response of the mixture as a whole, rather than two separate responses of each phase [14]. The drift flux model, despite being less rigorous than other more detailed two-phase flow models, is extremely important since it allows to properly predict and identify the physical structure of the flow in a relatively simple way [15]. Due to the importance of parameters such as the mixture velocity or pressure drop in the O\&G industry, several efforts have been made to improve the existing knowledge on drift velocity, and therefore improving the tools available to predict accurately multiphase flow behavior in pipelines.

\section{Materials and Methods}

The following section is divided in two main subsections: the experimental study carried out to complement and validate the information obtained from the computational simulations and the CFD modelling proposed for the simulations, which includes the spatial discretization constructed for the mesh and the physical models considered.

\subsection{Experimental Procedure}

The drift velocity was estimated experimentally by measuring the time it takes for the gas bubble to travel across a designated distance. The apparatus used consisted of a set of 3 acrylic pipes with equal longitudinal dimensions and varying diameters. All pipes were 2 meters long and had inner diameters of 17, 24 and $44 \mathrm{~mm}$, respectively. The lower end of the pipe was fitted with a plug and held by a highly resistant insulating tape to facilitate the filling process and guarantee that the plug would not yield under the fluid's weight. The pipe was set at four different inclination angles $\left(10^{\circ}, 20^{\circ}, 30^{\circ}\right.$ and $40^{\circ}$ ) with an adjusting metallic clamp and held horizontally on a previously calibrated stand at zero degrees of inclination with the upper end set above of a disposal bucket and fitted with an easily removable plug.

The measuring procedure consisted of one calibrated chronometer set to start as soon as the upper end plug was released. Once the bubble tip reached the marks previously placed on the tubes, the chronometer would be paused, and time would be recorded. All acrylic pipes were marked with the distances considered for the measurements, these distances being $0.5,1$ and $1.5 \mathrm{~m}$ for low viscosity fluids, and 0.2, 0.4, 0.5, 0.6, 0.8, 1 and $1.5 \mathrm{~m}$ for high viscosity Newtonian and non-Newtonian fluids. A general diagram of the experimental facility is shown in Fig. 1. The properties of the fluids considered in the present study are shown in Table 1, both for the CFD and the experimental tests. The Non-Newtonian fluids considered in the present study were modelled considering a power law to describe their rheological behavior with consistency index $K_{P}$ and flow behavior index $n_{P}$ [16]. As seen in Table 1, all Non-Newtonian fluids tested follow a pseudo-plastic behavior with a flow behavior index of $n_{P}<1$. In order to calculate the effective viscosity appropriately, the shear rate's definition was taken from Darby (2001) [17] as shown in Eq. (2).

$$
\mu_{e f f}=K_{P}(\dot{\gamma})^{n_{P}-1}=K_{p}\left(\frac{8 v_{d}}{D}\right)^{n_{p}-1}
$$

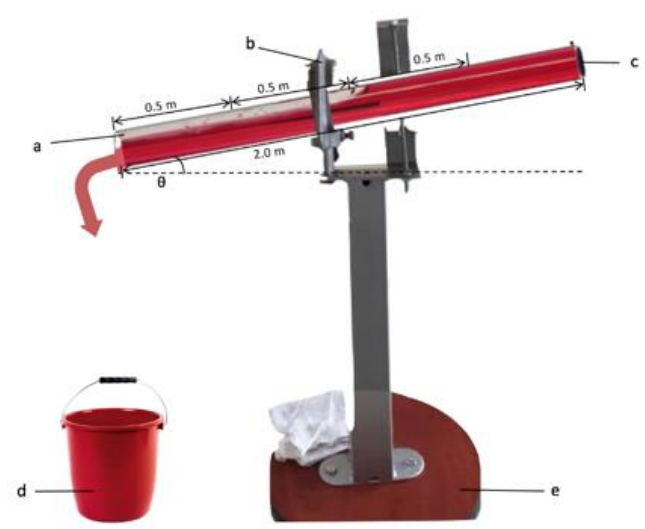

Fig. 1: Experimental facility. a. Penetrating gas bubble b. Adjustable metallic clamp c. Plug d. Bucket e. Base. 
Table 1: Measured properties of the fluids tested at 0.74 bar and $25^{\circ} \mathrm{C}$.

\begin{tabular}{cccccc}
\hline Fluid & $\boldsymbol{\rho}\left(\frac{\boldsymbol{k g}}{\boldsymbol{m}^{\mathbf{3}}}\right)$ & $\boldsymbol{\mu}(\mathbf{P a} * \mathbf{s})$ & $\boldsymbol{\sigma}\left(\frac{\mathbf{N}}{\mathbf{m}}\right)$ & Newtonian & Non-Newtonian \\
\hline Water & 1000.000 & 0.001 & 0.072 & $\mathrm{X}$ & \\
IsoparL Oil & 762.290 & 0.001 & 0.024 & $\mathrm{X}$ & \\
Mineral Oil & 863.104 & 0.031 & 0.032 & $\mathrm{X}$ & \\
Sunflower Oil & 899.190 & 0.057 & 0.033 & $\mathrm{X}$ & \\
Olive Oil & 924.391 & 0.067 & 0.032 & $\mathrm{X}$ & \\
Hydraulic Oil & 893.122 & 0.092 & 0.031 & $\mathrm{X}$ & \\
Maple Syrup & 1380.374 & 0.572 & 0.050 & $\mathrm{X}$ & \\
Honey & 1468.687 & 7.105 & 0.054 & $\mathrm{X}$ & $\mathrm{X}$ \\
Shampoo & 1001.460 & $\mathrm{~K}_{\mathrm{P}}=8.2122 ; \mathrm{n}_{\mathrm{P}}=0.716$ & 0.021 & & $\mathrm{X}$ \\
Chocolate Syrup & 1333.391 & $\mathrm{~K}_{\mathrm{P}}=26.597 ; \mathrm{n}_{\mathrm{P}}=0.319$ & 0.032 & & \\
\hline
\end{tabular}

\subsection{CFD Modelling}

The CFD simulations were performed in the commercial software STAR-CCM+ v12.04.011 considering a finite volume approach. The geometry that was simulated consisted of half a circular pipe with a symmetry plane, as shown in Fig 2. The boundary conditions as shown in Fig. 2 correspond to the surfaces of the pipe and the plug modelled as walls with no-slip condition and a pressure outlet with constant volume fraction of 1 for the gas phase. This pressure outlet will guarantee the entrance of the gas phase due to pressure difference into the pipe and the exit of the liquid phase. In this way, the penetration of the gas phase into the stagnant liquid within the tube will be appropriately modelled by the CFD simulation considered.

The spatial discretization of the pipeline was constructed considering a polyhedral volume meshing model and a prism layer model. The polyhedral mesh guarantees that each cell has a large number of immediate neighbouring cells of which the software can obtain information and use linear shape functions, resulting in a better approximation of the gradients, lower skewness angles and a more accurate flux calculation when compared to a tetrahedral mesh [18]. Additionally, a polyhedral shape allows a higher probability of finding a direction within the cell that aligns with the direction of the flow [18]. Moreover, according to several practical studies [19], polyhedral meshes need approximately four times fewer cells to achieve the same level of accuracy when compared to a tetrahedral mesh [18].

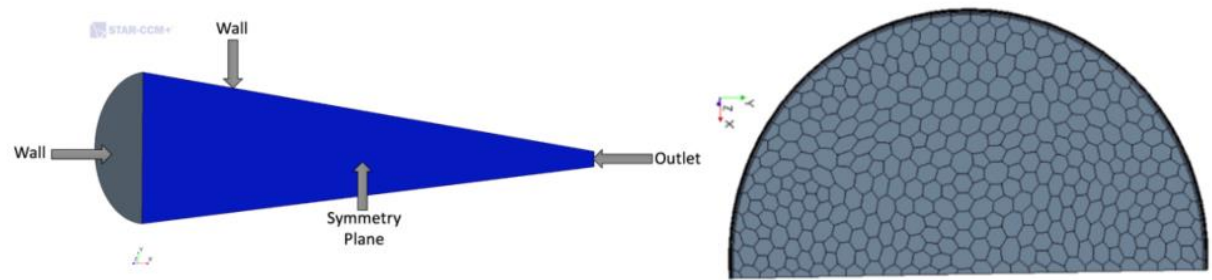

Fig. 2: Basic geometry of the pipeline and polyhedral mesh generated on the pipe.

The prism layer model allows the accurate resolution of near-wall flow features related to the boundary layer and the law of the wall on turbulent flow regimes [20]. These calculations are achieved by constructing prismatic orthogonal cells near a wall surface. This model additionally reduces a particular numerical discretization error near the wall boundary known as numerical diffusion. For this research, the number of prism layers was set to 8 with a constant growth rate of 1.5 to guarantee appropriate near-wall results. The prism layer total thickness was fixed as $24 \%$ relative to the base size of the volume mesh to assure an adequate $(<15)$ value of the dimensionless wall distance $(y+)$. This parameter was calculated for each case study applying the set of equations described in White, (2011) [21]. The final settings considered for the mesh construction are described in section 3.1 with the mesh independence test results.

The physics chosen to model the two-phase flow behavior on the current investigation was the Eulerian-Eulerian approach, implemented through the EMP and VOF models. These approaches allow a good interpretation of the physical phenomenon studied for the drift velocity, given that they consider appropriately the phase to phase interaction through the free surface flow and interphase tracking [22]. Additionally, these models allow the consideration of the mixture as a single pseudo-fluid rather than two separate phases by solving one mass-averaged momentum equation for the whole domain and 
one equation of continuity per phase to track the change on the interphase. Therefore, interphase/mixture parameters and variables can be calculated through interpolation using the volume fraction $\alpha_{k}$ of each phase per cell, as seen in Eqs (3) (4).

$$
\begin{gathered}
\frac{\partial \rho \overrightarrow{\mathbf{v}}}{\partial \mathrm{t}}+\nabla \cdot(\rho \overrightarrow{\mathbf{v}} \overrightarrow{\mathbf{v}})=-\nabla p+\nabla \cdot\left[\mu\left(\nabla \overline{\mathbf{v}}+\nabla \mathbf{v}^{\mathrm{T}}\right)\right]+\rho \overrightarrow{\mathbf{g}}+\overrightarrow{\mathbf{F}} \\
\frac{\partial \alpha_{\mathrm{k}}}{\partial \mathrm{t}}+\overrightarrow{\mathrm{v}} \nabla \alpha_{\mathrm{k}}=\frac{\mathrm{S}_{\alpha_{\mathrm{k}}}}{\rho_{\mathrm{k}}}
\end{gathered}
$$

The VOF model was selected because, for this application, it is especially useful for locating and accurately tracking the free surface region and therefore it can calculate correctly the volume fraction of the fluid on each cell [23]. The correct estimation of this parameter and the consideration of the surface tension forces allows the VOF model to predict accurately the flow pattern expected (slug flow). Moreover, this model also helps sharpening the interphase resolution between the liquid and gas phases [24], which reduces possible numerical diffusion [25]. The VOF method bases its approach on a single set of equations for the continuous phase, while the dispersed phase is modelled through an additional transport equation which takes into account its volume fraction [22].

Given that the two-phase flow phenomena for the drift flux is time dependent, an implicit unsteady study was considered. Density, viscosity (for Newtonian fluids) and surface tension values remain constant as energy transfer phenomenon is negligible and the drift velocities don't vary significantly in order of magnitude. This reduces the computational effort required to solve the equations of variation. Additionally, the segregated flow model was selected to numerically solve the flow equations in an uncoupled manner, which requires less memory and has a faster convergence rate [18]. This model has been selected for the study of multiphase flow in previous studies, as in Hernandez-Perez et al. (2010) [25], and has delivered satisfactory results.

The present study considers laminar regime flow for most of the fluids tested, given their associated Reynolds numbers, as will be mentioned in forthcoming sections. The only fluids which behave with a turbulent flow regime are water and IsoparL, for which a realizable two-layer $\kappa-\epsilon$ turbulence model was selected with all y+ wall treatment. The $\kappa-\epsilon$ turbulence model lies within the classification of RANS set of turbulence models, which allow to decompose the instantaneous variables in the N-S equations into their mean and their fluctuations. The realizable two-layer model was selected as it offers the most mesh flexibility, giving good results with fine low $y+$ meshes and producing the least inaccuracies for intermediate meshes (with y+ up to 30). Similarly occurs for the all y+ wall treatment, which was chosen as it offers the most mesh flexibility for wall spacing, guaranteeing the appropriate boundary layer resolution for coarser and fine meshes [18]. This turbulence model has been used in related studies [26] with satisfactory results.

\section{Results and Discussion}

The following section will be divided in 4 subsections: 1) Mesh independence test and global CFD and experimental comparison; 2) Drift flux behavior across the pipe's length; 3) Relationship between the drift flux and the angle of inclination and 4) Froude and Viscosity number relationship.

\subsection{Mesh Independence Test and Global CFD and Experimental Results Comparison}

To determine the optimal base size of the mesh for each case study considered (regarding viscosity, inclination, among other factors), various tests were performed as shown in Table 2. The mesh independence test for low-viscosity fluids was performed using four different fluids to assure a wide viscosity range, as seen in Table 2. On the other hand, only one high viscosity fluid was considered given the computational resources needed to perform these types of simulations. The results from the tests performed were compared with data reported in literature [10], [27] and experimental data gathered for the present study. A total of six base size values $(0.5 \mathrm{~cm}$ for mesh 'A', $0.25 \mathrm{~cm}$ for mesh 'B', $0.175 \mathrm{~cm}$ for mesh ' $\mathrm{C}$ ', $0.1 \mathrm{~cm}$ for mesh ' $\mathrm{D}$ ', $0.075 \mathrm{~cm}$ for mesh ' $\mathrm{E}$ ', $0.05 \mathrm{~cm}$ for mesh ' $\mathrm{F}$ ') were tested for low viscosity fluids. The high viscosity oil considered an additional set of three refined mesh sizes (base sizes of $0.075 \mathrm{~cm}$ for mesh 'A2', $0.05 \mathrm{~cm}$ for mesh 'B2' and $0.04 \mathrm{~cm}$ for mesh ' $\mathrm{C} 2$ '). 
Table 2: Mesh Independence Tests established.

\begin{tabular}{|c|c|c|c|c|c|c|c|}
\hline \multirow{3}{*}{$\begin{array}{l}\text { Fluid } \\
\text { Case }\end{array}$} & \multicolumn{6}{|c|}{ Low-viscosity } & \multirow{3}{*}{$\begin{array}{c}\text { High-viscosity } \\
\text { Generic Oil } 3 \\
-\end{array}$} \\
\hline & \multicolumn{2}{|c|}{ Water } & \multicolumn{2}{|c|}{ Mineral Oil } & Generic Oil 1 & Generic Oil 2 & \\
\hline & Case 1 & Case 2 & Case 1 & Case 2 & - & - & \\
\hline$\rho\left(\mathrm{kg} / \mathrm{m}^{3}\right)$ & \multicolumn{2}{|c|}{1000} & \multicolumn{2}{|c|}{836} & 884 & 869 & 1410 \\
\hline$\mu(\mathrm{Pa} * \mathrm{~s})$ & \multicolumn{2}{|c|}{0.001} & \multicolumn{2}{|c|}{0.034} & 0.342 & 0.104 & 6.12 \\
\hline$\sigma(\mathrm{N} / \mathrm{m})$ & \multicolumn{2}{|c|}{0.072} & \multicolumn{2}{|c|}{0.03} & 0.029 & 0.029 & 0.087 \\
\hline Pipe Diameter (m) & 0.0508 & 0.0508 & 0.044 & 0.044 & 0.0508 & 0.0508 & 0.0373 \\
\hline Inclination Angle $\left(^{\circ}\right)$ & 0 & 30 & 0 & 30 & 0 & 10 & 0 \\
\hline Distance travelled (m) & 0.5 & 0.5 & 0.5 & 0.5 & 0.5 & 0.5 & 0.25 \\
\hline Source of comparison & [27] & [27] & This study & This study & [27] & [27] & [10] \\
\hline
\end{tabular}

Fig. 3 shows the most representative results of the cases considered previously for drift velocity deviation and computational time in terms of the number of cells. As seen in Fig. 3(a), the 'A', 'B' and 'C' cases all generate simulations with low drift velocity errors and low computational time. The ' $B$ ' grid was selected for low viscosity horizontal fluids given that it has the lowest experimental error and a reasonable computational time. For the case of inclined pipelines with low-viscosity fluids (Fig. 3(b)), it can be seen that the mesh sizes ' $A$ ' and ' $C$ ' generate the lowest drift velocity errors. However, for the ' $A$ ' mesh size, the residuals observed showed significant oscillations, which may suggest an underlying convergence issue with the solution. Therefore, a more refined grid, case ' $\mathrm{C}$ ', was selected to guarantee more stable residuals and better convergence. For the high viscosity fluid, a similar case scenario was observed as for the inclined low viscosity fluids. The grid 'A2' had the lowest drift velocity deviation and computational time but oscillating residual values were observed for every iteration, which represents a poor convergence of the simulation. Consequently, the mesh size chosen for the simulations of high viscosity fluids was $0.05 \mathrm{~cm}$ ('B2'), to guarantee trustworthy results and a better convergence, despite the higher computational time consumed on each simulation.

(a)

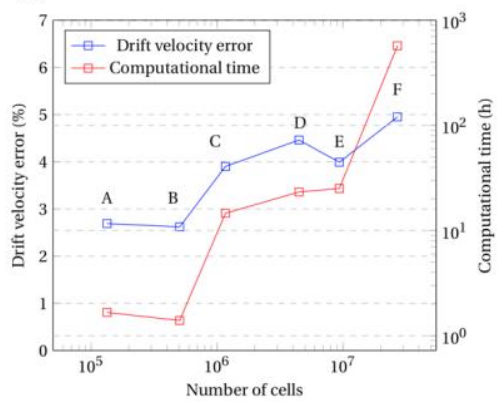

(b)

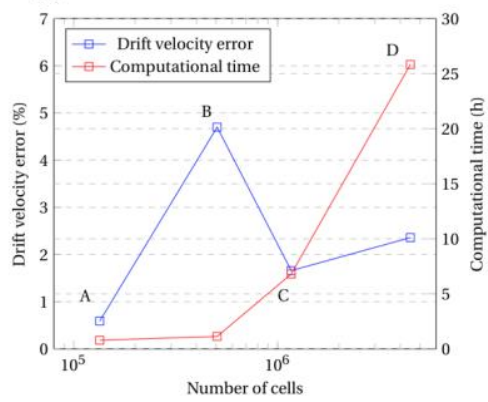

(c)

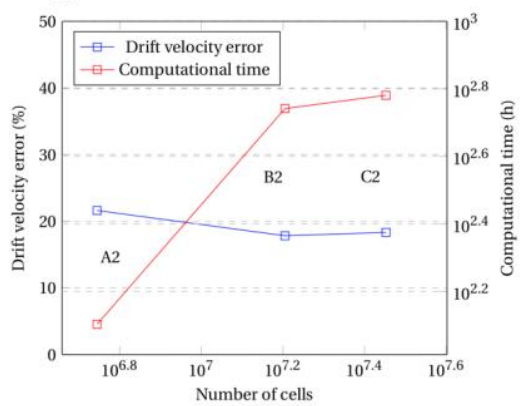

Fig. 3: Mesh Independence results for (a) Water on a horizontal setting (b) Water on a $30^{\circ}$ inclined setting (c) Generic Oil.

In the interest of comparing the CFD and the experimental data gathered on the present study, Fig. 4 (a) was constructed by plotting all the data available, considering all fluids and every different operational arrangement. In this figure, the great majority of the CFD data does not differ from its experimental counterpart by more than $30 \%$. In fact, Fig. 4 (b) shows that only around 5\% of the data surpasses a drift velocity deviation of $10 \%$ and that most of the simulations proposed predict a drift velocity with an error of about $0.22 \%$. From Fig. 4 (a) and Fig. 4 (b) it can also be noted that the highest drift velocity errors were observed for the lowest drift velocities, which translates into high viscosity fluids, as it was expected from the values observed on the mesh independence test. The CFD model proposed predicts accurately the drift flux behavior for low viscosity fluids in any operational arrangement but struggles to simulate appropriately this phenomenon for fluids with higher viscosities. In general, the CFD model tends to overestimate the viscous effect on the drift velocity, predicting slower velocities than the experimental data observed. Additionally, the CFD model fails to estimate correctly the surface tension effect of the IsoparL, as it will be seen in Fig. 6 the drift velocity measured experimentally is much higher than the predicted in CFD. Section 3.2 will show only CFD results given the good agreement observed with experimental data. 
(a)

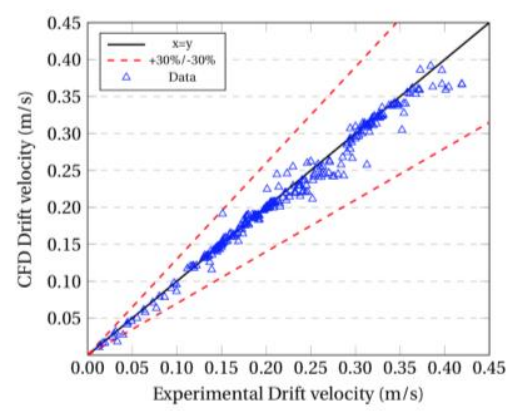

(b)

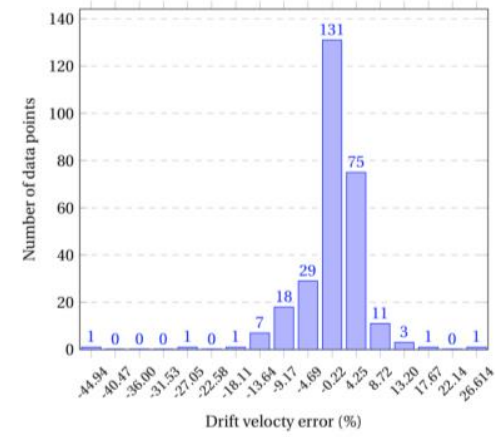

Fig. 4: (a) CFD vs. Experimental drift velocity for all the operating conditions and fluids tested. (b) Distribution of the error.

\subsection{Drift Flux Behavior across the Pipe'S Length}

From Fig. 5 (a) it can be appreciated that the drift velocity tends to decrease along the pipe's length more prominently as the viscosity of the liquid phase increases. This can be explained through the predominant forces on each fluid, considering the Reynolds number relationship between inertial and viscous forces. For Reynolds over 7000, corresponding to water and IsoparL Oil, the inertial forces are predominant over the viscous forces. This implies that there will be no significant obstruction for the penetration of the gas bubble, maintaining the drift velocity constant. On the other hand, for low Reynolds number under 500, the viscous forces will be predominant over the inertial. Therefore, these forces will represent a significant energetic barrier for the gas bubble to overcome as it travels along the pipe, which will cause the bubble to slow down. In contrast, Fig 5 (b) shows predominant inertial forces over the viscous for all fluids in the inclined setting, keeping the drift velocity constant along the pipe length except for very viscous fluids, as seen for maple syrup. As mentioned in the previous section, the CFD model tends to overestimate the viscous effect on the drift velocity for high viscosity fluids, which causes a higher descent of the drift velocity along the pipe's length compared to the experimental observations.

(a)

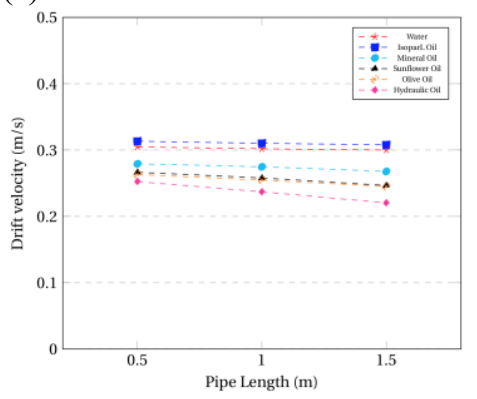

(b)

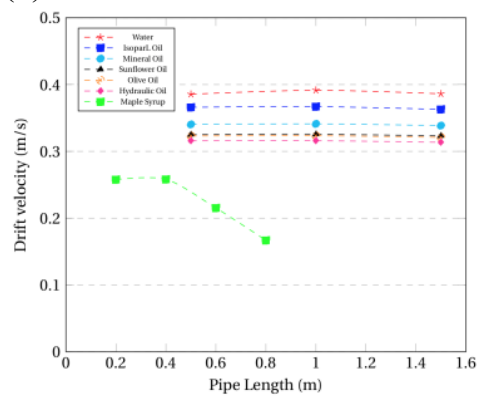

(c)

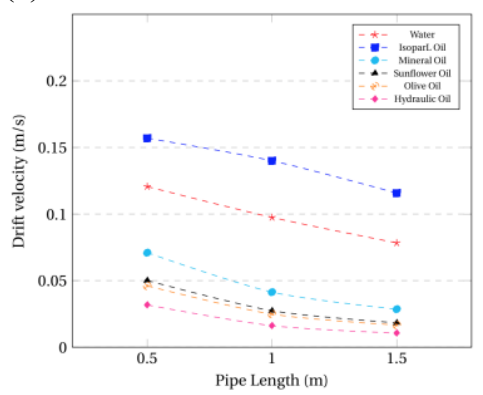

(d)

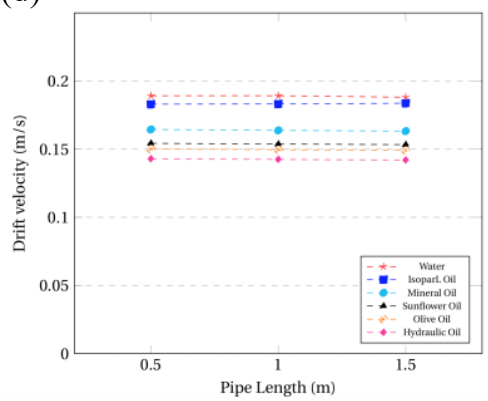

Fig. 5: CFD results for the drift velocity behavior across the pipe length for Newtonian Fluids in (a) $44 \mathrm{~mm}$ and $0^{\circ}$ (b) $44 \mathrm{~mm}$ and $40^{\circ}$ (c) $17 \mathrm{~mm}$ and $0^{\circ}$ (d) $17 \mathrm{~mm}$ and $40^{\circ}$.

A similar case is observed for Fig. 5 (c) and (d) but between capillary/surface tension and gravitational forces described by the Eötvos number. By calculating the Eö number as reported in literature [1], it can be observed that all fluids in the $17 \mathrm{~mm}$ pipe have values under 100, which corresponds to dominant capillary forces over gravitational [28]. On the contrary, all fluids on the $44 \mathrm{~mm}$ pipe have Eö numbers over 100, regardless of the fluid considered. In Fig. 5 (c) it can be appreciated that the drift velocity decreases considerably along the pipe's length for all fluids, regardless of their viscosity, opposite to the behavior observed for low viscosity fluids on the $44 \mathrm{~mm}$ pipe. This is due to the predominant capillary forces on the $17 \mathrm{~mm}$ pipe, which affect the penetration of the gas bubble on all fluids due to surface tension forces, slowing it down as it travels along the pipe. For the inclined configuration of the $17 \mathrm{~mm}$ pipe (Fig 5 (d)), it can be appreciated that the capillary forces are no longer significant on the system, as the drift velocity remains constant for all 
fluids, regardless of their viscosity and their Eö number. Therefore, inclined systems will have predominant inertial and gravitational forces.

\subsection{Relationship between the Drift Velocity and Inclination Angle}

The results obtained through the CFD model shown in Fig 6 (b) are in good agreement with the experimental results obtained by Moreiras (2014) [1] and Gockal (2008) [27]. From Fig 6 (a) and (b) the drift velocity tends to reach a plateau as the angle increases up to $40^{\circ}$, regardless of the viscosity of the fluid. This plateau is caused by the fact that the inertial forces become predominant over the viscous or capillary forces for higher inclination angles, as seen in the previous section. Therefore, as the viscous forces are less representative on the motion of the gas bubble, the drift velocity value will tend to be constant and will depend entirely on the inertia associated with the gas bubble's motion on each fluid. The only strong divergence to this pattern is the IsoparL Oil measured experimentally for the $40^{\circ}$ setting, as it keeps increasing almost linearly with increasing angle. This implies that the CFD simulation does not model appropriately the effects of surface tension for this oil, as previously discussed in section 3.1 .

(a)

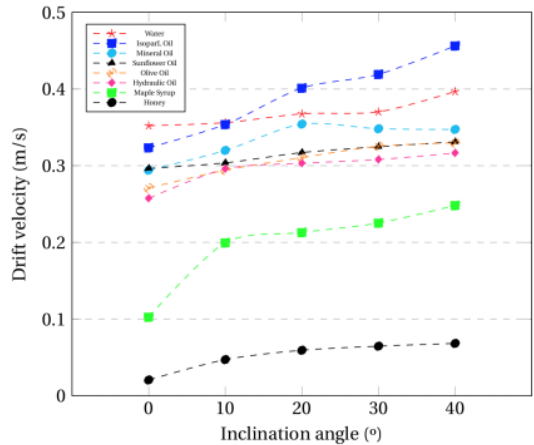

(b)

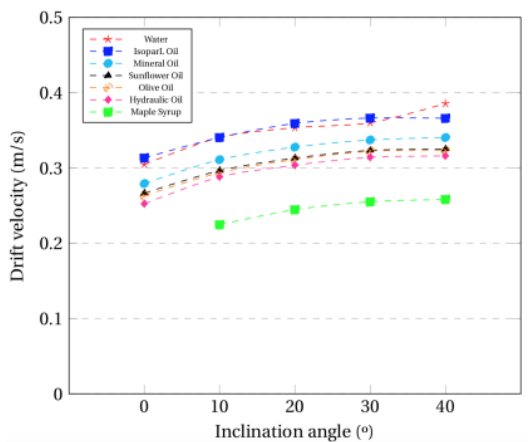

Fig. 6: Drift velocity behavior against the angle of inclination for Newtonian Fluids in (a) Experimental (b) CFD, considering a 44 mm pipe and a measurement at $0.5 \mathrm{~m}$ along the pipe's length.

\subsection{Froude Number vs Viscosity Number}

The calculation for the Froude and viscosity number was done following the equations established by Moreiras (2014) [1]. An inverse exponential relationship was observed for all inclination angles between the Fr and Nvis calculated at a distance of $0.5 \mathrm{~m}$ along the pipe, which is in good agreement with the results reported by Moreiras (2014) [1]. From Fig. 7 (a) it can be noted that, in general, the Fr calculated with the experimental drift velocity measured is slightly larger than those predicted by the CFD model. This observation accounts for the over estimation of the viscous effect on the CFD model as commented on previous sections, which results in lower drift velocities than the observed. The decrease of the Fr against the Nvis becomes less prominent as the inclination angle increases, which translates into the dominant inertial forces observed for high inclination angles over viscous or capillary forces. Additionally, it can be noted that the rate at which the Fr decreases against the Nvis shows a similar pattern between inclined pipelines, especially for high viscosity fluids (high Nvis). However, this rate of decrease changes significantly from a horizontal pipeline to an inclined one, as can be seen in Fig. 7 (b). 
(a)

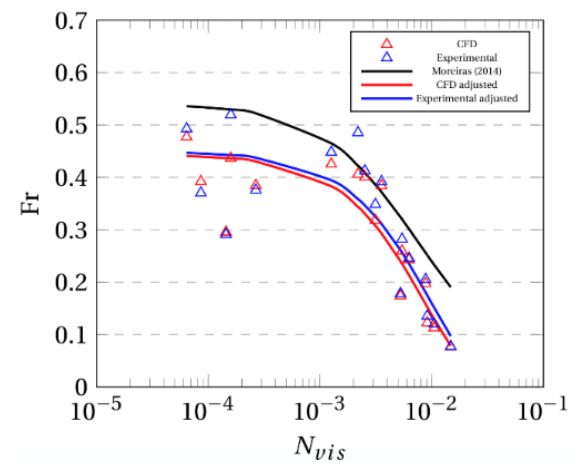

(b)

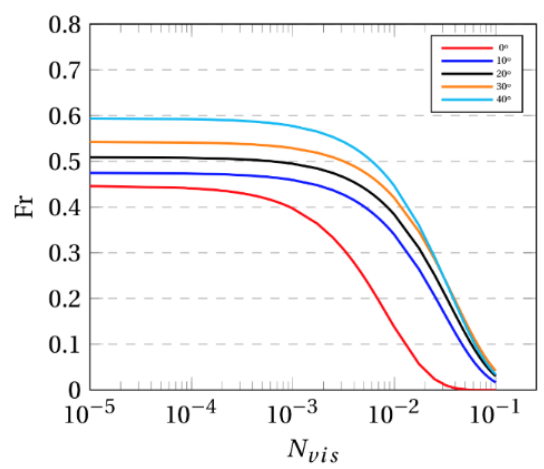

Fig. 7: (a) Froude vs Viscosity number at $0^{\circ}$ for Newtonian fluids, (b) Adjusted exponential function for Newtonian fluids (CFD).

From Fig. 8 it can be appreciated that Non-Newtonian fluids have smaller Fr numbers as compared to Newtonian fluids, given their high viscosity at low shear rates which occurs specially on a horizontal setting. Fig. 8 (a) and (b) shows a rapid decrease of the Fr for higher Nvis and smaller inclination angles. A drastic change in the Fr can be observed between the inclined pipelines and the horizontal setting, having smaller values by one order of magnitude. This behavior can be explained by the fact that, as opposed to inclined pipelines, the evacuation of the fluid on the horizontal case is caused only by the pressure difference between the initially sealed pipeline and the atmosphere, as there is no gravitational component influencing the motion of the gas bubble. Therefore, the shear rates occurring as the gas penetrates will be smaller to those on the inclined pipe, which implies that the effective viscosity of the liquid phase will be considerably larger. Consequently, the viscous forces that must be overcome are larger for the horizontal setting, resulting in smaller drift velocity values.

(a)

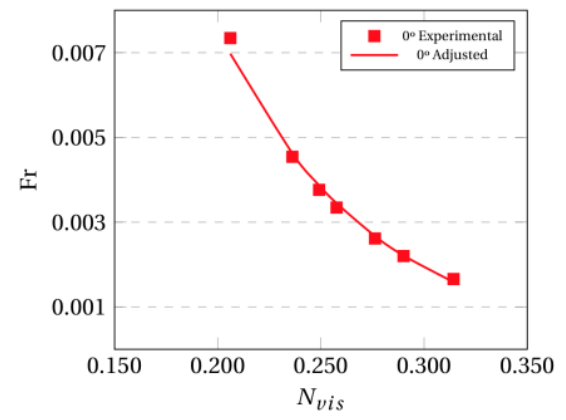

(b)

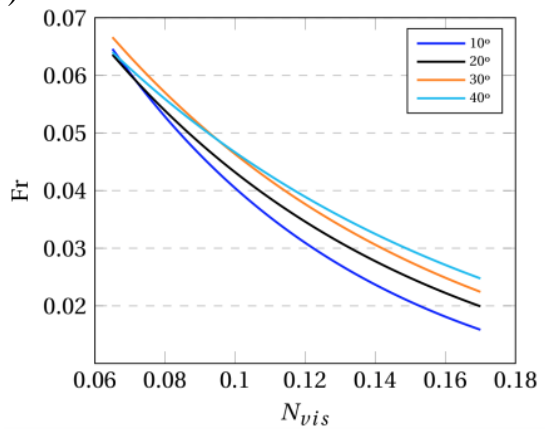

(c)

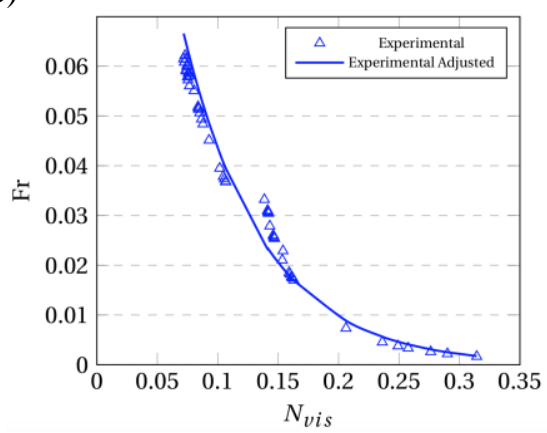

Fig. 8: (a), (b) Adjusted experimental exponential functions and (c) Fr vs Nvis experimental data for Non-Newtonian fluids.

Fig. 8 (c) shows the overall tendency between Fr and Nvis for all the experimental data gathered for Non-Newtonian fluids. It can be observed that this tendency may be interpreted as a continuation of the correlation suggested by Moreiras (2014) [1] for higher Nvis values, regardless of the operational settings considered. Even though it was observed that the rheological nature plays a fundamental role on the drift velocity measured, the Fr vs Nvis tendency observed for Newtonian fluids will still holds its relevancy for Non-Newtonian fluids with shear thinning characteristics.

\section{Conclusions}

The CFD modelling of the drift flux through different stagnant fluids at varying pipe diameters, lengths and inclination angles was the focus of the present study. From the results obtained, it can be first concluded that the proposed CFD model can correctly estimate the drift velocity behavior for low viscosity fluids and calculates it adequately for high viscosity fluids, maintaining deviations under $30 \%$. The drift velocity across the pipe's length tends to decrease for dominant capillary and viscous forces on Newtonian fluids, in which the condition of $E_{0}<100$ or $\operatorname{Re}<500$ was fulfilled and a horizontal setting was considered. However, for dominant inertial and gravitational forces, the drift velocity maintained a constant value along the pipe's length. For inclined configurations, it was found that, regardless the dominant forces, the 
drift velocity will always maintain a constant value. The behavior of the drift velocity against the angle of inclination showed a plateau region around $40^{\circ}$ degrees of inclination for all fluids, despite of their viscosity. This behavior was in good correspondence with the data reported in literature [1]. This plateau region corresponds to the shift of dominant forces, in which the viscous/capillary forces become less significant on the penetration of the gas bubble and the inertia associated with its motion governs the phenomena. Therefore, the drift velocity will maintain constant values for inclination angles around this plateau region.

As for the behavior of the Froude number against the viscosity number, it can be concluded that, for both, Newtonian and non-Newtonian fluids, the Froude number tends to decrease exponentially with an increase in the Viscosity number regardless of the operational conditions considered. This relationship is also in good agreement with results given in literature [1]. This decay becomes less prominent as the inclination angle increases. Therefore, it was found that the highest rate of change for the Froude number with the Viscosity number is observed at $0^{\circ}$ for all fluids tested in this study. This behavior was attributed to the fact that, when the pipeline is positioned completely horizontally, only the pressure gradient between the pipe and the atmosphere produces the evacuation of the liquid and the entry of the bubble, whereas, in inclined pipelines, the gravitational acceleration has an additional contribution which affects the dominant forces of the system and the rheological behaviour of Non-Newtonian fluids. It was concluded that the rheological nature of the fluids has an important influence on the behaviour of the drift velocity observed.

\section{References}

[1] J. Moreiras, E. Pereyra, C. Sarica, and C. F. Torres, "Unified drift velocity closure relationship for large bubbles rising in stagnant viscous fluids in pipes," J. Pet. Sci. Eng., vol. 124, pp. 359-366, 2014.

[2] R. D. Kaminsky, "Predicting single-phase and two-phase non-newtonian flow behavior in pipes," J. Energy Resour. Technol., vol. 120, no. 1, pp. 2-7, 1998.

[3] G. A. Gregory and D. S. Scott, "Correlation of liquid slug velocity and frequency in horizontal cocurrent gas-liquid slug flow," AIChE Journal, vol. 15, no. 6. pp. 933-935, 1969.

[4] A. E. Dukler and M. G. Hubbard, "A Model for Gas-Liquid Slug Flow in Horizontal and Near Horizontal Tubes," Ind. Eng. Chem. Fundam., vol. 14, no. 4, pp. 337-347, 1975.

[5] N. I. Heywood and J. F. Richardson, "Slug flow of air-water mixtures in a horizontal pipe: Determination of liquid holdup by $\gamma$-ray absorption," Chem. Eng. Sci., vol. 34, no. 1, pp. 17-30, 1979.

[6] T. B. Benjamin, "Gravity currents and related phenomena.," J. Fluid. Mech., vol. 31, no. 02, pp. 209-248, 1968.

[7] M. E. Weber, "Drift in intermittent two phase flow in horizontal pipes," The Canadian Journal of Chemical Engineering, vol. 59, no. 3. pp. 398-399, 1981.

[8] E. E. Zukoski, "Influence of viscosity, surface tension, and inclination angle on motion of long bubbles in closed tubes," J. Fluid Mech., vol. 25, no. 4, pp. 821-837, 1966.

[9] K. Bendiksen, "An Experimental Investigation of the Motion of Long Bubbles in Inclined Tubes," Int. J. Multiph. Flow, vol. 10, no. 4, pp. 467-483, 1984.

[10] M. E. Weber, A. Alarie, and M. E. Ryan, "Velocities of extended bubbles in inclined tubes," Chem. Eng. Sci., vol. 41, no. 9, pp. 2235-2240, 1986.

[11] D. J. Nicklin, M. A. Wilkes, and J. F. Davidson, “Two-Phase Flow in Vertical Tubes," Trans. Inst. Chem. Eng., vol. 40, pp. 61-67, 1962.

[12] R. F. Kroes and R. A. W. M. Henkes, "CFD for the motion of elongated gas bubbles in viscous liquid," BHR Gr. 9th North Am. Conf. Multiph. Technol. 2014, no. 1, pp. 283-298, 2014.

[13] Y. Taitel and D. Barnea, Encyclopedia of Two Phase Heat Transfer and Flow I. World Scientific Publishing Co., 2015.

[14] M. Ishii and T. Hibiki, Thermo-fluid dynamics of two-phase flow. Springer, 2006.

[15] L. Cheng, Frontiers and Progress in Multiphase Flow I. Springer, 2014.

[16] C. T. Crowe, Multiphase Flow Handbook, vol. 1218, no. 36. 2006.

[17] R. Darby, Chemical Engineering Fluid Mechanics, Revised and Expanded, vol. 2. New York: Taylor \& Francis, 2001.

[18] Siemens, "STAR CCM+ Documentation," Siemens. NY, 2017.

[19] M. Peric, "Polyhedral Grids In STAR-CCM+." CD-ADAPCO, pp. 1-31, 2017.

[20] R. B. Bird, W. E. Stewart, and E. N. Lightfoot, "Transport Phenomena," J. Wiley, 2007. 
[21] F. White, "Fluid Mechanics," McGraw-Hill,New York, p. 862, 2010.

[22] H. Pineda, T.-W. Kim, N. Ratkovich, and E. Pereyra, "CFD Modelling of High Viscosity Liquid-Gas Two-Phase Slug Flow," Universidad de los Andes, 2016.

[23] S. Jeon, S. Kim, and G. Park, "CFD simulation of condensing vapor bubble using VOF model," Proc. World Acad. Sci, vol. 3, no. 12, pp. 209-215, 2009.

[24] C. W. Hirt and B. D. Nichols, "Volume of fluid (VOF) method for the dynamics of free boundaries," J. Comput. Phys., vol. 39, no. 1, pp. 201-225, 1981.

[25] V. Hernandez-Perez, M. Abdulkadir, and B. Azzopardi, "Grid Generation Issues in the CFD Modelling of TwoPhase Flow in a Pipe," J. Comput. Multiph. Flows, vol. 3, no. 1, pp. 13-26, 2011.

[26] L. F. Barceló, P. A. Caron, A. E. Larreteguy, R. Gayoso, F. Gayoso, and G. Guido Lavalle, "Análisis del comportamiento de equipos separadores de agua-petróleo usando volumenes finitos y el modelo del drift flux," Mecánica Comput., vol. XXIX, pp. 8463-8480, 2010.

[27] B. Gockal, Q. Wang, H.-Q. Zhang, and C. Sarica, "Effects of High Oil Viscosity on Oil/Gas Flow Behavior in Horizontal Pipes," Soc. Pet. Eng. Proj. Facil. Constr., pp. 1-11, 2008.

[28] M. Ramdin and R. Henkes, "Computational Fluid Dynamics Modeling of Benjamin and Taylor Bubbles in TwoPhase Flow in Pipes," J. Fluids Eng., vol. 134, no. 4, p. 041303, 2012. 